\title{
Glioblastoma Presenting as Spontaneous Subdural Hematoma
}

\author{
Woowon $\mathrm{Oh}^{1}$, Yeongu Chung ${ }^{1}$, Jebeom Hong ${ }^{1}$, Yu Sam Won ${ }^{1}$, Pil-Wook Chung ${ }^{2}$, Myung Ho Rho ${ }^{3}$ \\ Departments of ${ }^{1}$ Neurosurgery, ${ }^{2}$ Neurology, ${ }^{3}$ Radiology, Kangbuk Samsung Hospital, Sungkyunkwan University School of Medicine, \\ Seoul, Republic of Korea \\ Corresponding author: Yu Sam Won \\ Department of Neurosurgery, Kangbuk Samsung Hospital, \\ Sungkyunkwan University School of Medicine, 29 Saemunan-ro, \\ Jongno-gu, Seoul 03181, Republic of Korea \\ Tel: $+82-2-2001-2159$ \\ Fax: $+82-2-2001-2157$ \\ A 76-year-old man presented with recurrent left side weakness for several \\ months without a history of head trauma. Brain computed tomography \\ revealed a subdural hematoma (SDH). The patient was diagnosed with \\ a chronic SDH and empirically treated via burr hole trephination. However, \\ the origin of SDH was eventually confirmed to be glioblastoma.
}

E-mail: yusam.won@samsung.com

Received: July 11, 2021

Revised: July 22, 2021

Accepted: July 23, 2021
Key Words: Glioblastoma; Hematoma, subdural; Hematoma, subdural, chronic

\section{INTRODUCTION}

Traumatic brain injury usually results from a violent blow to the head. Serious traumatic brain injury can result in a skull fracture, epidural hematoma, cerebral contusion, or subdural hematoma (SDH). A SDH is most often the result of trauma. Rarely, there are patients who suffer SDH without a definitive history of head trauma. Nontraumatic or spontaneous SDH is rare and many causes should be considered. The etiology of non-traumatic SDH includes cortical aneurysmal rupture, neoplasm, and spontaneous intracranial hypotension. In this report, we present a case of non-traumatic SDH caused by glioblastoma (GBM).

\section{CASE REPORT}

A 76-year-old man presented to another hospital with recurrent left side weakness that had begun several months prior without a history of head trauma. Brain computed tomography (CT) was performed and revealed acute and chronic mixed type SDH on the right convexity (Fig. 1). At that time, the medical team made a decision for the patient to be on conservative care, because the amount of SDH was subtle and the patient's symptoms were mild.

After one month, the patient's symptoms did not improve and left side weakness and hypoesthesia was aggravated; hence, the patient has transferred our hospital for further workup and management. Brain CT was performed and revealed SDH and new lowdensity lesion on the right frontoparietal area (Fig. 2). First, we assumed the newly observed lesion was a fluid collection from subdural hygroma or cerebral infarction. We expected the symptoms to be aggravated due to SDH and fluid collection and decided to perform surgical treatment via burr hole trephination for SDH drainage. After surgical management, a dark brownish fluid, a hallmark of typical chronic SDH, was drained. However, the amount of fluid drained was not significant and the patient's symptoms were not improved.

Postoperative brain CT showed a subtle reduction in SDH and a new high-density lesion at the site of the previous low-density

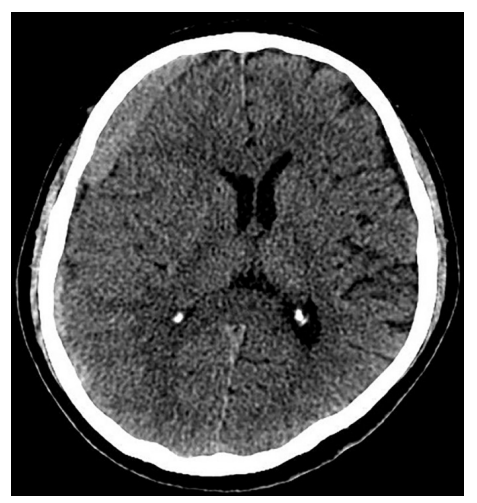

Fig. 1. Brain computed tomography scan shows mixed acute and chronic subdural hematoma in the right frontoparietal area.

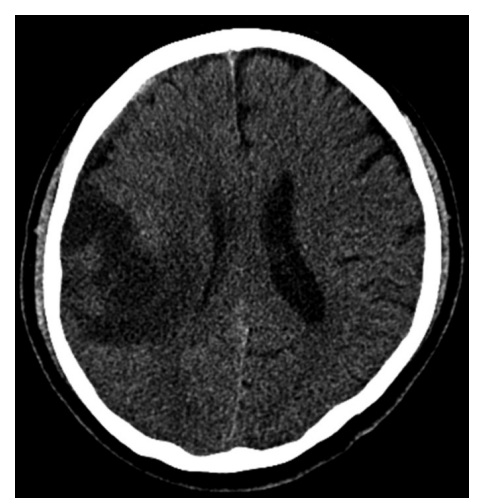

Fig. 2. Preoperative brain computed tomography scan reveals subdural hematoma and a low density lesion in the right frontoparietal area. 


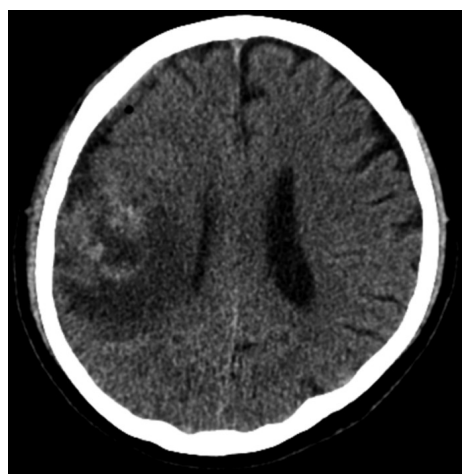

Fig. 3. Brain computed tomography scan after burr hole craniotomy shows a new high density lesion in the right frontoparietal area.
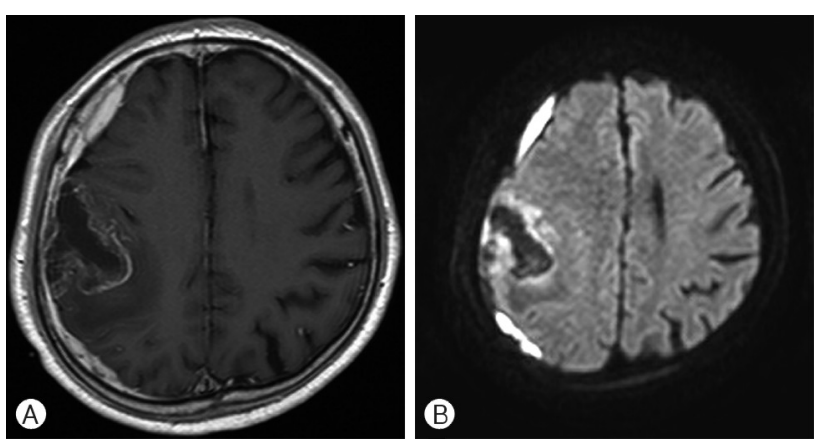

Fig. 4. (A) Brain magnetic resonance imaging (MRI) (T1-weighted image with enhancement, axial view) shows heterogeneous peripheral enhancing irregular mass in the right frontoparietal lobe. (B) Brain MRI (diffusion-weighted image, axial view) shows low signal intensity.

lesion (Fig. 3). For evaluation of this new lesion, a full-sequence brain magnetic resonance (MR) imaging (MRI) was performed. Brain MRI revealed a heterogeneous peripheral enhancing irregular masslike lesion with perilesional edema, for which we could not exclude brain tumor (Fig. 4). Craniotomy was performed for mass-lesion biopsy and removal. The result of mass-lesion biopsy was GBM.

The results of biopsy was GBM

Results of immunohistochemistry:

a) GFAP: positive in background

b) p53: strong and diffuse positivity

c) Ki-67: increased labeling index (about over 15\%)

d) CD34: increased vasculature with meshwork pattern

e) IDH-1: negative

GFAP: glial fibrillary acidic protein; IDH-1: isocitrate dehydrogenase 1.

Follow-up brain MRI examination showed the lesion was almost totally removed (Fig. 5). The subsequent concordant radiochemotherapy, which was initiated four weeks after surgery, was tolerated very well.

\section{DISCUSSION}

In general, patients with SDH have a history of head trauma.

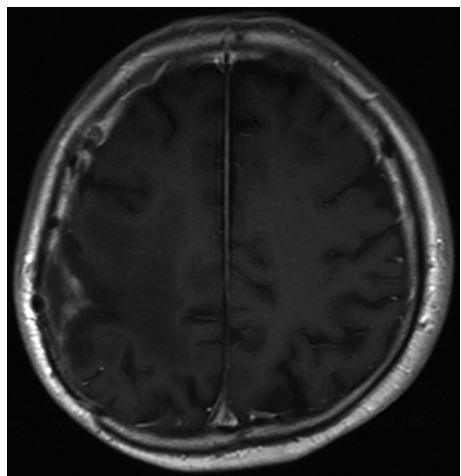

Fig. 5. Brain magnetic resonance imaging ( $\mathrm{T} 1$-weighted image with enhancement, oxial views) 1-year after surgical tumor resection followup shows stable glioblastoma status at the tumor resection site and no evidence of local tumor recurrence.

However, some SDHs without trauma history is observed. A broad cause of spontaneous SDH should be considered including vascular and even neoplastic origin. Coombs et al. ${ }^{5)}$ reported that among the $63 \%$ of cases where patient sex was specified, there was a 2:1 male predominance; overall, the majority of causes of spontaneous SDH were arterial (61.5\%), then idiopathic (10.8\%), coagulopathic (10.1\%), neoplastic (5.4\%), and spontaneous intracranial hypotension (5.4\%).

The known vascular origins of non-traumatic SDH are ruptures of small cortical arteries or intracranial aneurysms, rupture of arteriovenous malformation (AVM), and hypertensive or amyloid angiopathic intracerebral hematoma $(\mathrm{ICH})$ perforating the brain surface and arachnoid membrane $1,3,5,6,20,22)$. Aneurysmal rupture usually produces subarachnoid hemorrhage $(\mathrm{SAH})$ with or without $\mathrm{ICH}$ and/ or intraventricular hematoma (IVH). Aneurysmal SDH with or without $\mathrm{SAH}$ is rare, with an incidence estimated to be between $0.5 \%$ and $7.9 \%^{20)}$. A rare case of non-traumatic acute $\mathrm{SDH}$ without $\mathrm{SAH}$, which was caused by rupture of a cortical artery aneurysm, was reported and the patient did not have an infection, inflammation, neoplasm, or head traumal'. The pathogenesis of distal MCA or cortical artery aneurysms includes infectious or neoplastic emboli, arteritis, head trauma, and arterial dissection ${ }^{21)}$. In cases of AVM rupture, $\mathrm{ICH}$ is the most common, followed by IVH and SAH. Some authors reported rare cases of SDH due to rupture of cortical AVM and thought $A V M$ vessel adhesion to the arachnoid membrane and strain on the arachnoid membrane could have led to the formation of $\mathrm{SDH}$ rather than $\mathrm{SAH}$ or $\mathrm{ICH}^{3,6)}$. Rupture of a leptomeningeal artery adjacent to the dura, with extravasation into both the brain parenchyma and the subdural space, is a possible explanation of SDH and non-traumatic primary lobar $\mathrm{ICH}^{22)}$.

Spontaneous $\mathrm{ICH}$ is the most common bleeding complication in a patient with coagulopathy or on antiplatelet or anticoagulant therapy, followed by non-traumatic SDH or infrequent $\mathrm{SAH} \mathrm{H}^{2,7}$. Although the pathogenesis of non-traumatic $\mathrm{SDH}$ with coagulopathy or anticoagulant therapy remains unclear, many studies reported that the mortality rate in patients with acute or chronic SDH and coagulopathy was higher, ${ }^{7}$ )

Spontaneous intracranial hypotension $(\mathrm{SIH})$ is another well-known cause of non-traumatic SDH. SIH is sometimes associated with bilateral chronic SDH. The incidence of SDH among $\mathrm{SIH}$ patients ranges from $16 \%$ to $57 \%$, predominantly in males, whereas $\mathrm{SIH}$ 
without SDH predominantly occurs in females, ${ }^{8,14,18,30}$. Low cerebrospinal fluid pressure generates tears in the bridging veins of the dural border cell layer, followed by vein rupture ${ }^{5,25)}$.

Intracranial tumors are a well-known cause of hemorrhage into the brain parenchyma. Clinical and autopsy studies have reported that brain tumors were found in $0.9 \%$ to $11 \%$ of spontaneous $\mathrm{ICH}^{19,26,29)}$. However, non-traumatic SDH associated with neoplasm is believed to occur rarely and has several pathogenesis including hemorrhagic effusion from malignant tissue, dural vessel obstruction by neoplastic cells, angio-demoplastic reaction of the dura to tumor infiltration, and direct hematogenous metastasis of malignant tumor ${ }^{17,23,26,29}$. The incidence of brain hemorrhage from metastatic and primary intracranial malignant tumors is similar. Lung cancer" melanoma ${ }^{19)}$, and renal cell cancer ${ }^{28)}$, as well as choriocarcinoma ${ }^{24)}$, are implicated most frequently when brain hemorrhage results from a metastatic lesion. Oligodendroglioma or GBM primary intracra-

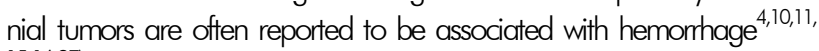
$15,16,27$. One retrospective study reported that the hemorrhage rate for mixed oligodendroglioma/astrocytoma was higher than $\mathrm{GBM}^{121}$.

Most SDHs are generally thought to be caused by head trauma and can happen in any age group. Brain CT scans are usually sufficient for diagnosis in cases of traumatic SDH. When a SDH patient presents without a history of head trauma, the differential diagnosis should include various causes, as mentioned above, and be evaluated via neuroimaging modalities including brain MRI and $C T$, and/or MR angiography in addition to complete medical history.

\section{CONCLUSION}

$\mathrm{SDH}$ is typically a sequela of head trauma; however, some cases without a definitive history of head trauma. So-called non-traumatic or spontaneous SDH can occur due to various causes like vascular lesions, neoplasms, coagulopathies, and $\mathrm{SIH}$.

When a patient suffers from SDH without a clear history of head trauma, it is wise to conduct additional examinations, such as CT angiography or brain MRI, to help determine the cause of SDH and to plan further treatment.

\section{CONFLICTS OF INTEREST}

No potential conflict of interest relevant to this article was reported.

\section{REFERENCES}

1. Awaji K, Inokuchi R, Ikeda R, Haisa T: Nontraumatic pure acute subdural hematoma caused by a ruptured cortical middle cerebral artery aneurysm: Case report and literature review. NMC Case Rep J 3:63-66, 2016

2. Butler AC, Tait RC: Management of oral anticoagulantinduced intracranial haemorrhage. Blood Rev 12:35-44, 1998

3. Choi HJ, Lee JI, Nam KH, Ko JK: Acute spontaneous subdural hematoma due to rupture of a tiny cortical arteriovenous malformation. J Korean Neurosurg Soc 58:547-549, 2015
4. Chrastina J, Novák Z, Riha I, Ghallab K: Primary brain tumor as a rare cause of acute subdural hematoma. Rozhl Chir 88: 549-553, 2009

5. Coombs JB, Coombs BL, Chin EJ: Acute spontaneous subdural hematoma in a middle-aged adult: case report and review of the literature. J Emerg Med 47:e63-e68, 2014

6. Datta NN, Chan KY, Kwok JC, Poon CY: Posterior fossa subdural hematoma due to ruptured arteriovenous malformation. Case report. Neurosurg Focus 8:ecp1, 2000

7. Hart RG, Boop BS, Anderson DC: Oral anticoagulants and intracranial hemorrhage. Facts and hypotheses. Stroke 26:14711477, 1995

8. Hashizume K, Watanabe K, Kawaguchi M, Fujiwara A, Furuya $\mathrm{H}$ : Evaluation on a clinical course of subdural hematoma in patients undergoing epidural blood patch for spontaneous cerebrospinal fluid leak. Clin Neurol Neurosurg 115:14031406, 2013

9. Ishii N, Yokosuka K, Sekihara Y, Hirano K, Suzuki Y, Ishii $\mathrm{R}$ : A case of subdural hematoma associated with dural metastasis of lung cancer. No Shinkei Geka 35:511-513, 2007

10. Kambe A, Hosoya T, Sakamoto M, Kondo S, Kurosaki M: High-grade glioma masquerading as a small cerebral hemorrhage: A case report. Yonago Acta Med 62:305-307, 2019

11. Kidoguchi M, Isozaki M, Hirose S, Kitai R, Kikuta KI: A case of subcortical intracerebral hemorrhage caused by underlying oligodendroglioma diagnosed through long-term follow-up. No Shinkei Geka 45:233-238, 2017

12. Kondziolka D, Bernstein M, Resch L, Tator CH, Fleming JF, Vanderlinden RG, et al.: Significance of hemorrhage into brain tumors: clinicopathological study. J Neurosurg 67:852-857, 1987

13. König SA, Schick U, Döhnert J, Goldammer A, Vitzthum HE: Coagulopathy and outcome in patients with chronic subdural haematoma. Acta Neurol Scand 107:110-116, 2003

14. Lai TH, Fuh JL, Lirng JF, Tsai PH, Wang SJ: Subdural haematoma in patients with spontaneous intracranial hypotension. Cephalalgia 27:133-138, 2007

15. Lee J, Kim MS, Kim YZ: Extensive pachymeningeal dissemination of glioblastoma mimicking chronic subdural hematoma: A case report. Brain Tumor Res Treat 7:39-43, 2019

16. Licata B, Turazzi S: Bleeding cerebral neoplasms with symptomatic hematoma. J Neurosurg Sci 47:201-210, 2003

17. Minette SE, Kimmel DW: Subdural hematoma in patients with systemic cancer. Mayo Clin Proc 64:637-642, 1989

18. Mokri B: Low cerebrospinal fluid pressure syndromes. Neurol Clin 22:55-74, vi, 2004

19. Navi BB, Reichman JS, Berlin D, Reiner AS, Panageas KS, Segal AZ, et al.: Intracerebral and subarachnoid hemorrhage in patients with cancer. Neurology 74:494-501, 2010

20. Nozar A, Philippe D, Fabrice P, Silvia M, Marc T: Acute pure spontaneous subdural haematoma from ruptured intracranial aneurysms. Interv Neuroradiol 8:393-398, 2002

21. Nussbaum ES, Madison MT, Goddard JK, Lassig JP, Nussbaum LA: Peripheral intracranial aneurysms: management challenges in 60 consecutive cases. J Neurosurg 110:7-13, 2009

22. Patel PV, FitzMaurice E, Nandigam RN, Auluck P, Viswanathan 
A, Goldstein JN, et al.: Association of subdural hematoma with increased mortality in lobar intracerebral hemorrhage. Arch Neurol 66:79-84, 2009

23. Reichman J, Singer S, Navi B, Reiner A, Panageas K, Gutin $\mathrm{PH}$, et al.: Subdural hematoma in patients with cancer. Neurosurgery 71:74-79, 2012

24. Rocque BG, Baskaya MK: Spontaneous acute subdural hematoma as an initial presentation of choriocarcinoma: a case report. J Med Case Rep 2:211, 2008

25. Schievink WI: Spontaneous spinal cerebrospinal fluid leaks and intracranial hypotension. JAMA 295:2286-2296, 2006

26. Schrader B, Barth H, Lang EW, Buhl R, Hugo HH, Biederer J, et al.: Spontaneous intracranial haematomas caused by neoplasms. Acta Neurochir (Wien) 142:979-985, 2000

27. Specht CS, Pinto-Lord C, Smith TW, DeGirolami U, Suran
E, Marshall PC, et al.: Spontaneous hemorrhage in a mixed glioma of the cerebellum: case report. Neurosurgery 19:278281, 1986

28. Spetzger U, Mull M, Sure U, Gilsbach J: Subarachnoid and intraventricular hemorrhage caused by hypernephroma metastasis, accompanied by innocent bilateral posterior communicating artery aneurysms. Surg Neurol 44:275-278, 1995

29. Wakai S, Yamakawa K, Manaka S, Takakura K: Spontaneous intracranial hemorrhage caused by brain tumor: its incidence and clinical significance. Neurosurgery 10:437-444, 1982

30. Yoon SH, Chung YS, Yoon BW, Kim JE, Paek SH, Kim DG: Clinical experiences with spontaneous intracranial hypotension: a proposal of a diagnostic approach and treatment. Clin Neurol Neurosurg 113:373-379, 2011 